\title{
Influence of Ponderomotive Potential and NonZero Initial Momentum of Ejected Electron on Transition Rate in Multiphoton Ionization
}

\author{
T.B. Miladinović* $*$ V.M. Petrović and V.M. Ristić \\ Department of Physics, Faculty of Science, Kragujevac University, Radoja Domanovića 12, Kragujevac, Serbia \\ (Received March 28, 2013; revised version May 14, 2013; in final form June 14, 2013)

\begin{abstract}
The multiphoton ionization of neutral atoms irradiated by photons is an interesting topic for experimental and
\end{abstract} \\ also theoretical examination. We study the influence of ponderomotive potential and non-zero initial momentum of \\ ejected electrons on transition rate, and also on the generalized cross-section in the case of a multiphoton ionization \\ process. Also, we study how the transition rate depends on the number of absorbed photons.
}

DOI: $10.12693 /$ APhysPolA.124.658

PACS: 32.80.-t, 32.80.Fb, 32.80.Wr

\section{Introduction}

Atoms and molecules generally can be ionized by different mechanisms. Some of mechanisms are tunnel and multiphoton ionization. To distinguish these two mechanisms it is convenient to introduce the Keldysh parameter [1], $\gamma$. This parameter is a measure of the adiabaticity, and is also called the adiabatic parameter. For an atom (or molecule) in a linearly polarized laser field with a field frequency $\omega$, the Keldysh parameter is defined as $\gamma=\frac{\omega}{e F} \sqrt{2 m E_{\mathrm{i}}}$, where $e$ is the electron charge, $E_{\mathrm{i}}$ - the ionization potential, $m$ - the electron mass, and $F$ is the strength of electromagnetic field. In atomic units, $e=m=\hbar=1$, which are used throughout this paper, the Keldysh parameter becomes $\gamma=\frac{\omega}{F} \sqrt{2 E_{\mathrm{i}}}$. The tunneling regime occurs when the parameter $\gamma$ has the values, $\gamma \ll 1$, while for values much greater than unity, $\gamma \gg 1$, the multiphoton regime dominates.

Since 1966, when the first multiphoton ionization experiments were performed by Voronov and Delone [2], many theoretical studies regarding different aspects of these processes were performed.

We theoretically investigated the transition rate and the generalized cross-section of an atom for the case of a multiphoton ionization process in a linearly polarized electromagnetic field by a plane wave laser field. We used a theoretical method based on the Ammosov-DeloneKrainov (ADK) theory [3]. Specifically, the influence of the ponderomotive potential and a non-zero initial momentum of an ejected electron on the aforementioned physical quantities was studied. Also, it was interesting to see how the number of absorbed photons influences the transition rate for some laser field intensities.

\section{Theoretical framework}

2.1. Transition rate without and with corrections

The multiphoton ionization process occurs in a strong laser field when the energy of a photon, $h \nu$, is not suffi-

*corresponding author; e-mail: tanja.miladinovic@gmail.com cient to overcome the binding energy of the valence electron. The valence electron is ejected from the bound state into the continuum only if the absorbed energy is higher than its binding energy.

In the framework of perturbation theory, the transition rate for ionization by a linearly polarized plane wave field can be calculated [4]:

$$
W \sim\left(\frac{F^{2} e}{8 \omega^{2} E_{\mathrm{i}}}\right)^{E_{\mathrm{i}} / \omega} .
$$

Here, it is assumed that the ponderomotive potential and the initial momentum of ejected electron were zero. In order to obtain more precise results for transition rates, it is necessary to improve the aforementioned formula (Eq. (1)).

First, we take into account the influence of the ponderomotive potential on the transition rate. The ponderomotive potential is time averaged kinetic energy of free electron oscillations in a laser field. The presence of the ponderomotive potential causes an increase of the ionization potential which means that more photons or a stronger laser field is necessary for the ionization process to occur.

When in the above expression we include the ponderomotive potential $U_{\mathrm{p}}$ :

$$
U_{\mathrm{p}}=\frac{F^{2}}{4 \omega^{2}},
$$

it obtains the following form:

$$
W_{U \mathrm{p}} \sim\left(\frac{F^{2} e}{8 \omega^{2}\left(E_{\mathrm{i}}+\frac{F^{2}}{4 \omega^{2}}\right)}\right)^{\frac{E_{\mathrm{i}}+\frac{F^{2}}{4 \omega^{2}}}{\omega}},
$$

where $W_{U \mathrm{p}}$ denotes the transition rate with the included ponderomotive potential.

Next, we consider the influence of a non-zero initial momentum of ejected electron on the transition rate. It is commonly assumed that the aforementioned momentum is equal to zero. But we find that the inclusion of non-zero initial momentum has an influence on transition rate.

In paper [5], the expression for the transition rate when the non-zero initial momentum of ejected electron is in- 
cluded, $W_{\mathrm{p}}$, was derived

$$
W_{\mathrm{p}} \sim\left(\frac{F^{2} e}{4 \omega^{2} p^{2}}\right)^{p^{2} / 2 \omega} .
$$

In order to generalize this expression, we need a formula for momentum expressed by known parameters such as $\omega, E_{\mathrm{i}}$, and $F$.

Let us recall that the energy conservation law [6] is

$$
K \omega=E_{\mathrm{i}}+U_{\mathrm{p}}+E_{\mathrm{k}},
$$

where $E_{\mathrm{k}}$ is the kinetic energy of an ejected electron whereas $K$ is the number of absorbed photons

$$
K=\left\langle\frac{E_{\mathrm{i}}}{\omega}+1\right\rangle ;
$$

here, the brackets $\langle\ldots\rangle$ mean the integer quotient of the number. It follows from Eq. (5) that the momentum, $p$, is

$$
p=\sqrt{2\left(K \omega-E_{\mathrm{i}}-\frac{F^{2}}{4 \omega^{2}}\right)} .
$$

An improved form of the formula for multiphoton ionization rate is obtained by combining Eq. (4) and Eq. (7):

$$
W_{\mathrm{p}} \sim\left(\frac{F^{2} e}{8 \omega^{2}\left(K \omega-E_{\mathrm{i}}-\frac{F^{2}}{4 \omega^{2}}\right)}\right)^{\left(K \omega-E_{\mathrm{i}}-\frac{F^{2}}{4 \omega^{2}}\right) / \omega} .
$$

\subsection{Generalized cross-section}

Multiphoton ionization process also can be described by a generalized cross-section:

$$
W_{K}=\sigma_{K} I^{K},
$$

where $\sigma_{K}$ is the $K$-photon generalized ionization cross-section and $I$ is the laser intensity.

Equation (9) presents the basic relation between transition rate and generalized cross-section. This dependence can also be expressed through the formula for a generalized cross-section [7] given in the following form:

$$
\sigma_{K}=\frac{8 \pi \omega W_{K}}{c F^{2}}
$$

Based on this equation we considered three cases of a generalized cross-section, $\sigma=\frac{8 \pi \omega W}{c F^{2}}, \sigma_{U \mathrm{p}}=\frac{8 \pi \omega W_{U_{\mathrm{p}}}}{c F^{2}}$ and $\sigma_{\mathrm{p}}=\frac{8 \pi \omega W_{\mathrm{p}}}{c F^{2}}$ expressed by transition rates defined in the text above, i.e. using Eq. (1), (3), and (8), respectively.

\section{Discussion}

In this paper we observed how transition rates and generalized cross-sections depend on the laser field intensity. We have also discussed the influence of the ponderomotive potential and initial momentum of ejected electron on the behavior of these two quantities.

Calculations were performed for atoms of the noble gas Ar. Atoms are ionized by a laser of $800 \mathrm{~nm}$ wavelength. The laser field was linearly polarized and intensity regime was between $5 \times 10^{11}$ and $3 \times 10^{13} \mathrm{~W} / \mathrm{cm}^{2}$. We used this intensity range because, for these intensities, the multiphoton ionization process is dominant. All graphs correspond to the particular value of $K$, i.e. to the minimal number of photons required for ionization process.

Let us first analyze the transition rate without any corrections (Eq. (1)) as a function of laser field intensity. This dependence is shown in Fig. 1.

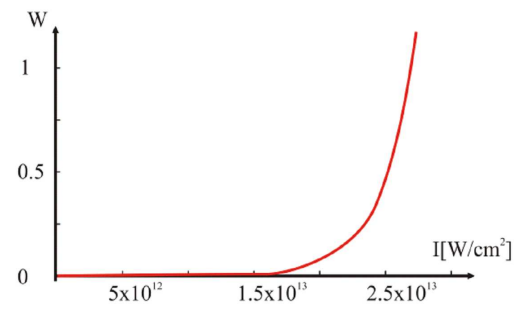

Fig. 1. The ionization rate, $W$, as a function of the laser field intensity, $I$.

The main conclusion that follows from this graph is that the transition rate does not have the expected Gaussian distribution form [8]. The reason for this deviation is that we neglected some of the quantities. So we included the ponderomotive potential in the formula for the transition rate. Based on the improved formula (Eq. (3)) Fig. 2 is obtained.

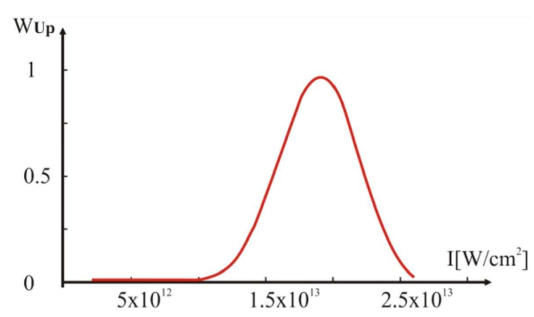

Fig. 2. The ionization rate, $W_{U \mathrm{p}}$, versus the field intensity, $I$.

The dependence shown in Fig. 2 has a Gaussian form. It is easily noticeable that ionization probability is increasing (for certain values of the external field) and decreasing, when the external field is higher. The maximal value of transition rate is for $I=1.9 \times 10^{13} \mathrm{~W} / \mathrm{cm}^{2}$.

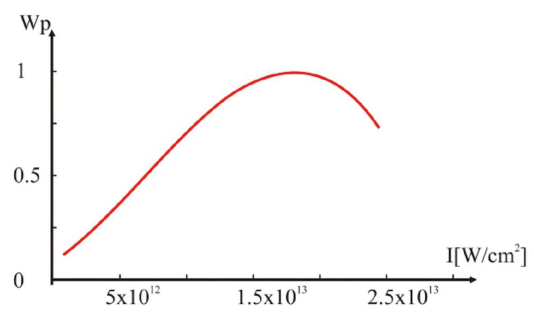

Fig. 3. The transition rate, $W_{\mathrm{p}}$, with the non-zero initial momentum and the ponderomotive potential corrections included.

In Fig. 3, we show the transition rate with both the ponderomotive potential and the initial momentum of 
an ejected electron included as a function of the driving field intensity.

In this case the maximal value of probability is for $I=1.95 \times 10^{13} \mathrm{~W} / \mathrm{cm}^{2}$.

By comparing this value with the value for the case when there is only the ponderomotive potential included, we can see that this maximal value is moved to the right a bit, i.e. to higher values of laser intensity. This shift occurs because part of the laser field energy is used to overcome ponderomotive potential, leaving smaller amounts of light energy available for ionization of remaining electrons.

Next, we give a short note about the behavior of the generalized cross-section. We considered the case without corrections of the transition rate and for the cases when the corrections are involved. As the result we obtained Fig. 4.

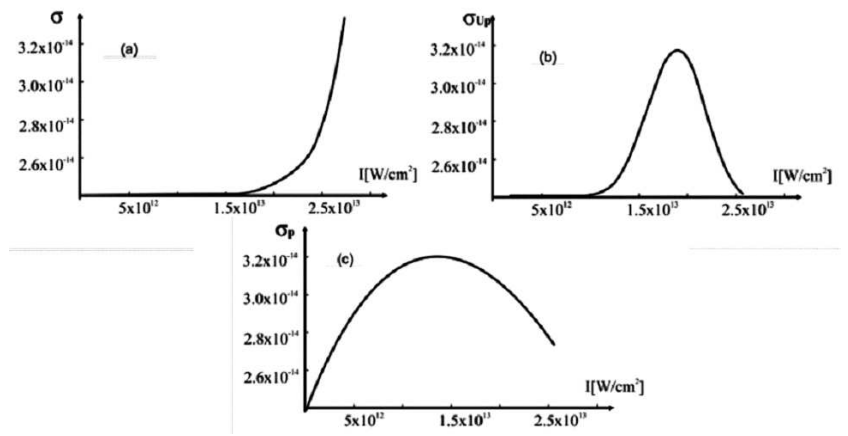

Fig. 4. Generalized cross-section when we used transition rate (a) without corrections, $W$, (b) with correction for ponderomotive potential, $W_{\mathrm{Up}}$, (c) with correction for non-zero initial momentum, $W_{\mathrm{p}}$.

It follows from this figure that the behavior of the generalized cross-section is in complete accordance with the behavior of the transition rate for the same conditions.

As we already noted it is interesting to see how the number of absorbed photons influences the transition rate. Also, in Fig. 5b, we show how the transition rate changes as a function of the number of absorbed photons and the laser field intensity.

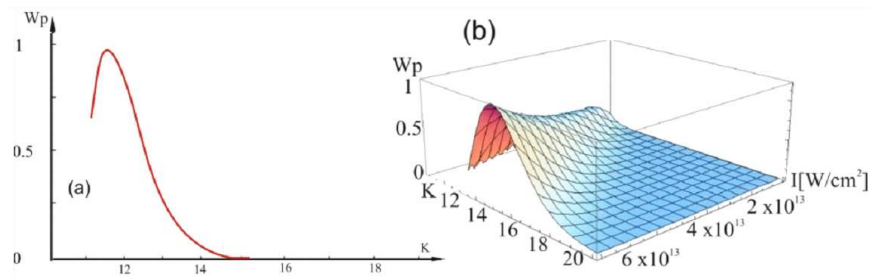

Fig. 5. (a) Dependence of the transition rate on the number of absorbed photons for $I$ fixed at $2 \times 10^{13} \mathrm{~W} / \mathrm{cm}^{2}$, (b) dependence of the transition rate on the number of absorbed photons and the laser field intensity.
In Fig. 5, it can be clearly seen that the transition rate is equal to zero when the number of absorbed photons is less than 11 because this is the minimum number of photons required (see Eq. (6)) for the ionization of an electron in a valence shell of Ar. When the number of absorbed photons increases, then the transition rate increases, until some maximal value, and after that decreases, keeping a constant value and asymptotically approaching the axis.

Figure 5b shows that the transition rate and the number of absorbed photons increase with an increasing laser field intensity.

\section{Conclusion}

In summary, we have studied the transition rate and the generalized cross-section for the multiphoton ionization process of an argon atom exposed to a linearly polarized laser field.

As mentioned earlier, it was often assumed that ponderomotive potential is negligible and that ejected electrons are leaving the atom with zero initial momentum. These assumptions forced us to examine the influence on transition rate of the multiphoton ionization. Our observations have shown that the maximum of the transition rate moves to the right, i.e. to higher values of laser field intensity, when the respective corrections are taken into account. One more reason for the study of transition rates is because the transition rate and the ion yield are in direct proportion. The fact is that the ion yield is more often measured in experiments and examination of transition rates can give useful information when analyzing the experimental results [9].

\section{Acknowledgments}

We are grateful to the Serbian Ministry of Education and Science for financial support through projects 171020 and 171021.

\section{References}

[1] L.V. Keldysh, Sov. Phys. JETP 20, 1307 (1965).

[2] G.S. Voronov, N.B. Delone, Zh. Eksp. Teor. Fiz. 50, 78 (1966); Sov. Phys. JETP 23, 54 (1966).

[3] V.M. Ammosov, N.B. Delone, V.P. Krainov, Sov. Phys. JETP 64, 1191 (1986).

[4] V. Ristić, Ph.D. Thesis, Kragujevac University, Moscow-Kragujevac 1991.

[5] D.I. Bondar, Phys. Rev. A 78, 015405 (2008).

[6] N.B. Delone, V.P. Krainov, Physics-Usp. 41, 469 (1998).

[7] N.B. Delone, V.P. Krainov, in: Multiphoton Processes in Atoms, 2nd ed., Springer, New York 2000.

[8] N.B. Delone, V.P. Krainov, Physics-Usp. 38, 1247 (1995).

[9] M.D. Perry, O.L. Landen, A. Szöke, E.M. Campbell, Phys. Rev. A 37, 747 (1988). 